\title{
Análise de Escores de Risco para Predição de Mortalidade em Pacientes Submetidos à Cirurgia Cardíaca por Endocardite
}

\author{
Analysis of Risk Scores to Predict Mortality in Patients Undergoing Cardiac Surgery for Endocarditis
}

Fernando Pivatto Júnior, ${ }^{10}$ Clarissa Carmona de Azevedo Bellagamba, ${ }^{1}$ Eduardo Gatti Pianca, ${ }^{1}$ Fernando Schmidt Fernandes, ${ }^{1}$ Maurício Butzke, ${ }^{10}$ Stefano Boemler Busato, ${ }^{1}$ Miguel Gus ${ }^{1}$

Hospital de Clínicas de Porto Alegre, 1 Porto Alegre, RS - Brasil

\section{Resumo}

Fundamento: Escores de risco estão disponíveis para uso na prática clínica diária, mas saber qual deles escolher é ainda incerto.

Objetivos: Avaliar o EuroSCORE logístico, o EuroSCORE II e os escores específicos para endocardite infecciosa STSIE, PALSUSE, AEPEI, EndoSCORE e RISK-E na predição de mortalidade hospitalar de pacientes submetidos à cirurgia cardíaca por endocardite ativa em um hospital terciário de ensino do sul do Brasil.

Métodos: Estudo de coorte retrospectivo incluindo todos os pacientes com idade $\geq 18$ anos submetidos à cirurgia cardíaca por endocardite ativa no centro do estudo entre 2007 e 2016 . Foram realizadas análises de calibração (razão de mortalidade observada/esperada, O/E) e de discriminação (área sob a curva ROC, ASC), sendo a comparação das ASC realizada pelo teste de DeLong. $\mathrm{P}<0,05$ foi considerado estatisticamente significativo

Resultados: Foram incluídos 107 pacientes, sendo a mortalidade hospitalar de 29,0\% (IC95\%: 20.4-37.6\%). A melhor razão de mortalidade O/E foi obtida pelo escore PALSUSE (1,01, IC95\%: 0,70-1,42), seguido pelo EuroSCORE logístico (1,3, IC95\%: 0,92-1,87). O EuroSCORE logístico apresentou o maior poder discriminatório (ASC 0,77), significativamente superior ao EuroSCORE II $(p=0,03)$, STS-IE $(p=0,03)$, PALSUSE $(p=0,03)$, AEPEI $(p=0,03)$ e RISK-E $(p=0,02)$.

Conclusões: Apesar da disponibilidade dos recentes escores específicos, o EuroSCORE logístico foi o melhor preditor de mortalidade em nossa coorte, considerando-se análise de calibração (mortalidade O/E: 1,3) e de discriminação (ASC 0,77). A validação local dos escores específicos é necessária para uma melhor avaliação do risco cirúrgico. (Arq Bras Cardiol. 2020; 114(3):518-524)

Palavras-chave: Procedimentos Cirúrgicos Cardiovasculares/mortalidade; Endocardite/complicações; Mortalidade Hospitalar; Medição de Risco.

\begin{abstract}
Background: Risk scores are available for use in daily clinical practice, but knowing which one to choose is still fraught with uncertainty.

Objectives: To assess the logistic EuroSCORE, EuroSCORE II, and the infective endocarditis (IE)-specific SCOres STS-IE, PALSUSE, AEPEI, EndoSCORE and RISK-E, as predictors of hospital mortality in patients undergoing cardiac surgery for active IE at a tertiary teaching hospital in Southern Brazil.

Methods: Retrospective cohort study including all patients aged $\geq 18$ years who underwent cardiac surgery for active IE at the study facility from 2007-2016. The scores were assessed by calibration evaluation (observed/expected [O/E] mortality ratio) and discrimination (area under the ROC curve [AUC]). Comparison of AUC was performed by the DeLong test. A $p<0.05$ was considered statistically significant.

Results: A total of 107 patients were included. Overall hospital mortality was 29.0\% (95\%Cl: 20.4-37.6\%). The best O/E mortality ratio was achieved by the PALSUSE SCore (1.01, 95\%Cl: 0.70-1.42), followed by the logistic EuroSCORE (1.3, 95\%Cl: 0.92-1.87). The logistic EuroSCORE had the highest discriminatory power (AUC 0.77), which was significantly superior to EuroSCORE II $(p=0.03)$, STS-IE ( $p=0.03)$, PALSUSE $(p=0.03)$, AEPEI $(p=0.03)$, and RISK-E $(p=0.02)$.

Conclusions: Despite the availability of recent IE-specific scores, and considering the trade-off between the indexes, the logistic EuroSCORE seemed to be the best predictor of mortality risk in our cohort, taking calibration (O/E mortality ratio: 1.3) and discrimination (AUC 0.77) into account. Local validation of IE-specific scores is needed to better assess preoperative surgical risk. (Arq Bras Cardiol. 2020; 114(3):518-524)
\end{abstract}

Keywords: Cardiovascular Surgical Procedures/mortality; Endocarditis/complications; Hospital Mortality; Risk Assessment.

Full texts in English - http://www.arquivosonline.com.br

Correspondência: Fernando Pivatto Júnior •

Hospital de Clínicas de Porto Alegre - Rua Ramiro Barcelos, 2350. CEP 90035-903, Porto Alegre, RS - Brasil

E-mail: fpivatto@gmail.com

Artigo recebido em 22/01/2019, revisado em 19/04/2019, aceito em 03/06/2019

DOI: https://doi.org/10.36660/abc.20190050 


\section{Introdução}

Apesar dos avanços no tratamento, a endocardite infecciosa (EI) está associada a morbidade e mortalidade significativas. ${ }^{1}$ A correção cirúrgica da El ativa está associada à maior taxa de mortalidade dentre todas as doenças valvares, com taxas globais de mortalidade hospitalar acima de $20 \%{ }^{2}$

A cirurgia é atualmente realizada em 50 a $60 \%$ dos pacientes com El. ${ }^{3}$ As indicações são: insuficiência cardíaca (geralmente relacionada à disfunção valvar), infecção não controlada (geralmente associada à extensão perivalvular e a defeitos de condução atrioventricular), e prevenção da embolia sistêmica. ${ }^{4}$ Embora essas indicações sejam claras, a sua aplicação prática baseia-se em grande parte na condição clínica, nas comorbidades, e no risco operatório do paciente. ${ }^{5}$

Modelos de predição de risco para cirurgia cardíaca vêm sendo desenvolvidos para fornecer informações acerca dos riscos tanto para os médicos quanto para os pacientes, bem como para guiar a tomada de decisão. ${ }^{6} \mathrm{~A}$ avaliação do risco cirúrgico ajuda a medir a qualidade do serviço de saúde, e o perfil de risco é essencial para diferenciar os pacientes de acordo com a gravidade da condição de saúde. Do mesmo modo, conhecer o risco do paciente pode permitir a implementação de estratégias individualizadas, visando prevenir complicações. ${ }^{7}$ Embora haja escores de risco disponíveis para uso na prática clínica diária, ainda há muita incerteza sobre qual deles escolher. Nesse contexto, o objetivo deste estudo foi avaliar o EuroSCORE logístico, ${ }^{8}$ o EuroSCORE II ${ }^{9}$ e escores específicos para a EI, STS-IE, ${ }^{2}$ PALSUSE, ${ }^{10}$ AEPEI, ${ }^{11}$ EndoSCORE ${ }^{7}$ e RISK-E, ${ }^{12}$ como preditores de mortalidade hospitalar em pacientes submetidos à cirurgia cardíaca em razão de El ativa, em um hospital universitário terciário da região sul do Brasil.

\section{Métodos}

Este estudo de coorte retrospectivo incluiu todos os pacientes com $\geq 18$ anos de idade que foram submetidos à cirurgia cardíaca em razão de El ativa, no Hospital de Clínicas de Porto Alegre (HCPA), de 2007 a 2016. Apenas pacientes com El definitiva diagnosticada com base nos critérios de Duke modificados ${ }^{13}$ foram incluídos. Os pacientes foram identificados através dos agendamentos cirúrgicos e da busca por palavras-chave no sistema eletrônico de registros médicos do HCPA. O presente estudo foi aprovado pelo Comitê de Ética em Pesquisa da instituição (protocol no 16-0632).

O risco cirúrgico pré-operatório foi calculado através da média do EuroSCORE ${ }^{8}$ logístico e do EuroSCORE II, ${ }^{9}$ além dos escores específicos STS-IE, ${ }^{2}$ PALSUSE, ${ }^{10}$ AEPEI, ${ }^{11}$ EndoSCORE ${ }^{7}$ e RISK-E' ${ }^{12}$ (Tabela 1). A mortalidade durante a internação, independente do tempo de permanência, foi definida como mortalidade hospitalar. O clearance de creatinina (CC) foi estimado através da fórmula de Cockcroft-Gault. ${ }^{14}$

A insuficiência renal aguda foi definida como qualquer um dos seguintes critérios: aumento $\geq$ a $0,3 \mathrm{mg} / \mathrm{dL}$ da creatinina em 48 horas ou aumento de $\geq 1,5$ vezes da creatinina em relação ao valor basal, conhecido ou que se presume nos 7 dias anteriores; volume urinário
$<0,5 \mathrm{~mL} / \mathrm{kg} / \mathrm{h}$ em 6 horas. ${ }^{15} \mathrm{O}$ estado crítico no préoperatório foi definido como a presença de qualquer uma das caracteríticas a seguir durante a mesma internação hospitalar que a da cirurgia: taquicardia/fibrilação ventricular ou morte súbita recuperada; massagem cardíaca; ventilação ventilação mecânica antes da sala anestésica; administração de inotrópicos; uso de balão de contrapulsação/dispositivo de assistência ventricular antes da sala anestésica ou insuficiência renal aguda (anúria ou oligúria $<10 \mathrm{~mL} / \mathrm{h}$ ). ${ }^{9} \mathrm{~A}$ El ativa (ainda com tratamento antibiótico no momento da cirurgia), doença pulmonar crônica, arteriopatia extracardíaca, mobilidade reduzida (comprometimento grave de mobilidade secundária à disfunção neuromúsculo-esquelética), infarto do miocárdio recente ( $\leq 90$ dias), hipertensão arterial pulmonar grave (pressão arterial pulmonar sistólica $>55 \mathrm{mmHg}$ ), disfunção renal grave $(\mathrm{ClCr}<50 \mathrm{~mL} / \mathrm{min})$, e urgência de cirurgia também foram definidas conforme o EuroSCORE II. ${ }^{9}$

\section{Análise Estatística}

Os dados foram coletados diretamente dos prontuários eletrônicos dos pacientes e analisados utilizando os softwares IBM SPSS 21.0, MedCalc 12.5 e OpenEpi 3.01. ${ }^{16}$ Os dados qualitativos foram exibidos por meio de frequência absoluta e relativa; média (desvio padrão) ou mediana (intervalo interquartil) foram utilizadas para as análises quantitativas. A normalidade da distribuição de cada variável foi avaliada por meio do teste Shapiro-Wilk. A calibração (expressa pela razão entre a mortalidade observada e a esperada [O/E], isto é, a razão de mortalidade padronizada [RMP]) e a capacidade discriminatória (expressa pela área sob a curva ROC [ASC]) dos escores foi avaliada. Para calcular a RPM com intervalo de confiança (IC) de 95\%, utilizamos o teste exato de Mid-p modificado por Miettinen. A comparação da ASC foi realizada pelo teste de DeLong. Valores de $p<0,05$ foram considerados estatisticamente significantes.

\section{Resultados}

Durante o período estudado, foram incluídos 107 pacientes submetidos à cirurgia cardíaca na instituição durante a fase aguda da El. A idade média dos pacientes foi de 58,1 \pm 14,5 anos, sendo $24,3 \%$ mulheres. A El aórtica isolada foi a forma predominante de EI (43,9\%). As características dos pacientes e os detalhes cirúrgicos estão descritos na Tabela 2.

O tamanho mediano das vegetações foi de 14,0 mm (9,25$18,0)$. Trinta e um pacientes $(29,0 \%)$ apresentaram pelo menos 1 evento embólico, diagnosticado com base nos sintomas ou incidentalmente: $13(12,1 \%)$ para o sistema nervoso central e $11(10,3 \%)$ para o baço. Vinte e dois (20,6\%) estavam em diálise no pré-operatório: 14 (13,1\%) devido à doença renal crônica, 6 (5,6\%) devido à insuficiência renal aguda, e 2 (1,9\%) devido à doença renal crônica agudizada.

A cirurgia foi realizada com um tempo médio de 12,5 $(6,0-22,25)$ dias do início da antibioticoterapia. A principal indicação para cirurgia foi insuficiência cardíaca (76,6\%). O procedimento mais frequentemente realizado foi a troca valvar aórtica mecânica ( $\mathrm{n}=26 ; 24,3 \%$ ), seguida da troca 
Tabela 1 - Escores específicos para endocardite infecciosa analisados no presente estudo

\begin{tabular}{|c|c|c|c|c|}
\hline \multicolumn{5}{|c|}{ ESCORES NÃO ESPECÍFICOS } \\
\hline \multicolumn{2}{|c|}{ EuroSCORE, $1999^{8}$} & & \multicolumn{2}{|c|}{ EuroSCORE II, $2012^{9}$} \\
\hline \multicolumn{2}{|c|}{ Endocardite ativa } & & \multicolumn{2}{|c|}{ Endocardite ativa } \\
\hline \multicolumn{2}{|r|}{ Idade } & & \multicolumn{2}{|l|}{ Idade } \\
\hline \multicolumn{2}{|c|}{ Estado crítico no pré-operatório } & & \multicolumn{2}{|c|}{ Angina CCS classe 4} \\
\hline \multicolumn{2}{|c|}{ Cr sérica > $200 \mu \mathrm{mol} / \mathrm{L}$} & & \multicolumn{2}{|c|}{ Doença pulmonar crônica } \\
\hline \multicolumn{2}{|c|}{ Arteriopatia extracardiaca } & & \multicolumn{2}{|c|}{ Estado crítico no pré-operatório } \\
\hline \multicolumn{2}{|c|}{ Sexo feminino } & & \multicolumn{2}{|c|}{ Arteriopatia extracardíaca } \\
\hline \multicolumn{2}{|r|}{ FEVE } & & \multicolumn{2}{|c|}{ Sexo feminino } \\
\hline \multicolumn{2}{|c|}{ Disfunção neurológica } & & \multicolumn{2}{|l|}{ DMID } \\
\hline \multicolumn{2}{|c|}{ Cirurgia não coronariana } & & \multicolumn{2}{|l|}{ FEVE } \\
\hline \multicolumn{2}{|c|}{ Doença pulmonar } & & \multicolumn{2}{|c|}{ Classe funcional (NYHA) } \\
\hline \multicolumn{2}{|c|}{ Cirurgia cardíaca prévia } & & \multicolumn{2}{|c|}{ Mobilidade reduzida } \\
\hline \multicolumn{2}{|c|}{ IAM recente } & & \multicolumn{2}{|c|}{ Cirurgia cardíaca prévia } \\
\hline \multicolumn{2}{|c|}{ PSAP > $60 \mathrm{mmHg}$} & & \multicolumn{2}{|c|}{ IAM recente } \\
\hline \multicolumn{2}{|c|}{ Cirurgia na aorta torácica } & & Disfunção re & \\
\hline & ina instável & & PSAP & \\
\hline & Jrgência & & Cirurgia na aorta & \\
\hline Rupt & septo ventricular & & Urgência & \\
\hline & & & Procedimento re & \\
\hline & & CORES ESPECÍFICOS PARA & & \\
\hline STS-IE, $2011^{2}$ & PALSUSE, $2014^{10}$ & AEPEI, $2017^{11}$ & EndoSCORE, $2017^{12}$ & RISK-E, $2017^{13}$ \\
\hline Endocardite ativa & El de valva protética & $\mathrm{IMC}>27 \mathrm{Kg} / \mathrm{m}^{2}$ & Idade & Insuficiência renal aguda \\
\hline Arritmia* & Idade & $\begin{array}{l}\text { Estado crítico no } \\
\text { pré-operatório }\end{array}$ & DPOC & Idade \\
\hline Choque cardiogênico & $\begin{array}{l}\text { Grande destruição } \\
\text { intracardíaca }^{\dagger}\end{array}$ & $\mathrm{eTFG}<50 \mathrm{~mL} / \mathrm{min}$ & Cr sérica $\geq 2 \mathrm{mg} / \mathrm{dL}$ & Choque cardiogênico \\
\hline Doença pulmonar crônica & Staphylococcus spp. & Classe Funcional IV (NYHA) & Sexo feminino & Complicações perianulares ${ }^{\ddagger}$ \\
\hline Hipertensão sistêmica & Cirurgia urgente & PSAP > $55 \mathrm{mmHg}$ & FEVE & El de valva protética \\
\hline DMID/DMNID & Sexo (feminino) & & $\begin{array}{l}\text { Número de valvas/ } \\
\text { próteses tratadas }\end{array}$ & Choque séptico \\
\hline Procedimento valvar múltiplo & EuroSCORE $\geq 10 \%$ & & $\begin{array}{l}\text { Microorganismo isolado } \\
\text { na hemocultura }\end{array}$ & Trombocitopenia§ \\
\hline $\begin{array}{l}\text { Inotrópicos ou BIA no } \\
\text { pré-operatório }\end{array}$ & & & Presença de abscesso & Microrganismo virulento" \\
\hline CRM prévia & & & & \\
\hline Cirurgia valvar prévia & & & & \\
\hline $\begin{array}{l}\text { Insuficiência Renal (HD) ou } \\
\text { Cr sérica }>2 \mathrm{mg} / \mathrm{dL}\end{array}$ & & & & \\
\hline Urgência & & & & \\
\hline
\end{tabular}

*Taquicardia ventricular sustentada, fibrilação ventricular, fibrilação atrial, flutter atrial ou bloqueio atrioventricular de terceiro grau. ${ }^{\dagger}$ Abscessos ou outros achados ecocardiográficos sugestivos de infecção invasiva (comunicação entre câmaras, dissecção da parede ou extensa deiscência da válvula). ${ }^{\ddagger}$ Abscesso, pseudoaneurisma, fístula ou deiscência protética.

$\S<150.000$ plaquetas $/ \mathrm{mm}^{3}$. I/Staphylococcus aureus ou fungos. IMC: índice de massa corporal; CRM: cirurgia de revascularização miocárdica; CCS: Canadian Cardiovascular Society; DPOC: doença pulmonar obstrutiva crônica; Cr: creatinina; eTFG: estimativa da taxa de filtração glomerular; HD: hemodiálise; DMID: diabetes mellitus insulino-dependente; DMNID: Diabetes mellitus não insulino-dependente; El: endocardite infecciosa; FEVE: fração de ejeção do ventrículo esquerdo; IAM: infarto agudo do miocárdio; NYHA: New York Heart Association; PSAP: pressão sistólica da artéria pulmonar. 


\section{Artigo Original}

\section{Tabela 2 - Características dos pacientes e variáveis cirúrgicas}

\begin{tabular}{|c|c|}
\hline VARIÁVEL & $\mathrm{n}=107$ \\
\hline Idade (anos) & $58,1 \pm 14,5$ \\
\hline Sexo feminino & $26(24,3)$ \\
\hline Hipertensão arterial sistêmica & $60(56,1)$ \\
\hline Classe funcional III/IV (NYHA) & $53(49,5)$ \\
\hline Abscesso valvar & $40(37,4)$ \\
\hline Cirurgia cardíaca prévia & $35(32,7)$ \\
\hline Doença valvar degenerativa & $31(29,0)$ \\
\hline HAP grave & $31(29,0)$ \\
\hline Endocardite protética & $31(29,0)$ \\
\hline Insuficiência renal aguda & $30(28,0)$ \\
\hline Disfunção renal grave* & $25(26,0)$ \\
\hline Diálise & $22(20,6)$ \\
\hline Trombocitopenia & $20(18,7)$ \\
\hline Estado crítico no pré-operatório & $19(17,8)$ \\
\hline FEVE $\leq 50 \%$ & $17(15,9)$ \\
\hline DMID & $14(13,1)$ \\
\hline Endocardite infecciosa prévia & $11(10,3)$ \\
\hline Valvulopatia reumática & $10(9,3)$ \\
\hline Válvula aórtica bicúspide & $8(7,5)$ \\
\hline Arteriopatia extracardíaca & $8(7,5)$ \\
\hline IAM prévio & $8(7,5)$ \\
\hline Doença pulmonar crônica & $7(6,5)$ \\
\hline Mobilidade reduzida & $7(6,5)$ \\
\hline IAM recente & $3(2,8)$ \\
\hline Angina CCS classe 4 & $1(0,9)$ \\
\hline \multicolumn{2}{|l|}{ Localização da endocardite infecciosa } \\
\hline Valva aórtica & $47(43,9)$ \\
\hline Valva mitral & $35(32,7)$ \\
\hline Valvas aórtica + mitral & $20(18,7)$ \\
\hline Valva tricúspide & $4(3,7)$ \\
\hline Valvas tricúspide + mitral & $1(0,9)$ \\
\hline Microrganismo causador identificado & $72(67,3)$ \\
\hline Streptococcus viridans & $19(17,8)$ \\
\hline Enterococcus sp. & $10(9,3)$ \\
\hline Staphylococcus aureus & $9(8,4)$ \\
\hline \multicolumn{2}{|l|}{ Magnitude da intervenção } \\
\hline Outra que não CRM isolada & $81(75,7)$ \\
\hline Dois procedimentos & $25(23,4)$ \\
\hline Três procedimentos & $1(0,9)$ \\
\hline \multicolumn{2}{|l|}{ Urgência } \\
\hline Urgente & $98(91,6)$ \\
\hline Emergente & $9(8,4)$ \\
\hline CRM associada & $8(7,5)$ \\
\hline Tempo de circulação extracorpórea (min) & $84,0(65,0-110,0)$ \\
\hline Tempo de isquemia (min) & $65,0(51,0-84,0)$ \\
\hline \multicolumn{2}{|c|}{$\begin{array}{l}\text { CRM: cirurgia de revascularização do miocárdio; CCS: Canadian Cardiovascular } \\
\text { Society; DMID: diabetes mellitus insulino-dependente; NYHA: New York Heart } \\
\text { Association; HAP: hipertensão arterial pulmonar; FEVE: fracãa de ejeção do } \\
\text { ventrículo esquerdo; IAM: infarto agudo do miocárdio. *Excluímos pacientes } \\
\text { submetidos à hemodiálise no pré-operatório }(n=22 ; 20,6 \%) \text { e aqueles cujos } \\
\text { dados de peso corporal não estavam disponiveis }(n=11 ; 10,3 \%) \text {, o que tornou } \\
\text { impossivel calcular o clearance de creatinina. Dados expressos como média } \\
\text { desvio padrão, } n(\%) \text {, ou mediana (intervalo interquartill). }\end{array}$} \\
\hline
\end{tabular}

valvar aórtica biológica $(\mathrm{n}=22 ; 20,6 \%)$ e da troca valvar mitral biológica $(n=22 ; 20,6 \%)$.

A mortalidade hospitalar global foi de 29,0\% (IC95\%: 20,4$37,6 \%)$. Houve uma grande variação na mortalidade esperada dentre os escores, de 10,0\% no EndoSCORE a 28,6\% no escore PALSUSE (Figura 1). A melhor razão de mortalidade O/E foi obtida pelo escore PALSUSE (1,01; IC95\%: 0,701,42; $p=0,919)$, seguido pelo EuroSCORE logístico $(1,3$; IC95\%: 0,92-1,87; $p=0,123)$, conforme mostrado na Tabela 3. Todos os outros escores subestimaram significativamente a mortalidade hospitalar.

O EuroSCORE logístico teve o maior poder discriminatório (ASC: 0,77), como pode ser visto na Tabela 3 , sendo significativamente superior ao do EuroSCORE II $(p=0,03)$, STS-IE $(p=0,03)$, PALSUSE $(p=0,03)$, AEPEI $(p=0,03)$, e RISK-E $(p=0,02)$, embora não significativamente quando comparado com o EndoSCORE $(p=0,90)$. Todas as outras comparações foram não significativas, exceto EndoSCORE vs. AEPEI $(p=0,03)$.

\section{Discussão}

Nesta coorte de pacientes submetidos à cirurgia cardíaca devido a El, a melhor razão de mortalidade O/E e poder discriminatório foram obtidos pelo escore PALSUSE $(1,01)$ e pelo EuroSCORE (ASC: 0,77), respectivamente. O EuroSCORE logístico, que apresentou a segunda melhor razão O/E $(1,3)$, apresentou poder discriminatório significativamente melhor do que o escore PALSUSE (ASC: 0,$68 ; p=0,03$ ).

A ASC, também conhecida como estatística C ou índice C, é um marcador de precisão diagnóstica global ${ }^{17} \mathrm{e}$ uma medida efetiva e combinada de sensibilidade e especificidade..$^{18} \mathrm{O}$ poder discriminatório é considerado excelente se $>0,80$, muito bom se $>0,75$, e bom (aceitável) se $>0,70$. Além disso, avaliamos a calibração utilizando a razão de mortalidade O/E. Idealmente, essa razão é 1 , isto é, a mortalidade observada é igual à mortalidade esperada, indicando um modelo preditivo perfeitamente calibrado. Um valor de $\mathrm{O} / \mathrm{E}>1$ significa que o modelo subestimou a mortalidade, ao passo que um valor $<$ 1 significa que o modelo superestimou a mortalidade. Se o IC de $95 \%$ para a razão de mortalidade O/E cruza 1, o modelo está bem calibrado. ${ }^{19}$ Contudo, é possível que um modelo de risco tenha boa calibração, mas pouca discriminação, e viceversa. A discriminição é mais importante que a calibração; um modelo pode ser recalibrado ou reajustado conforme a prática for aprimorada, porém se o modelo for construído levando em conta fatores de risco incorretos, sua discriminação não poderá ser melhorada..$^{20}$ Embora o EndoSCORE não tenha apresentado um poder discriminatório significativamente pior quando comparado com o EuroSCORE logístico, ele subestimou significativamente a mortalidade hospitalar; desse modo, a realização de ajustes seria necessária. Na nossa coorte, o EuroSCORE logístico demonstrou ser o melhor preditor de risco de mortalidade.

O microrganismo causador foi identificado em apenas $67,3 \%$ dos casos nesta coorte, diferentemente das coortes de validação dos escores específicos para $\mathrm{El}$, nas quais a taxa de detecção foi de $81,0-86,6 \% .{ }^{10-12}$ De modo semelhante, o Staphylococcus aureus, que causa uma infecção agressiva e, 


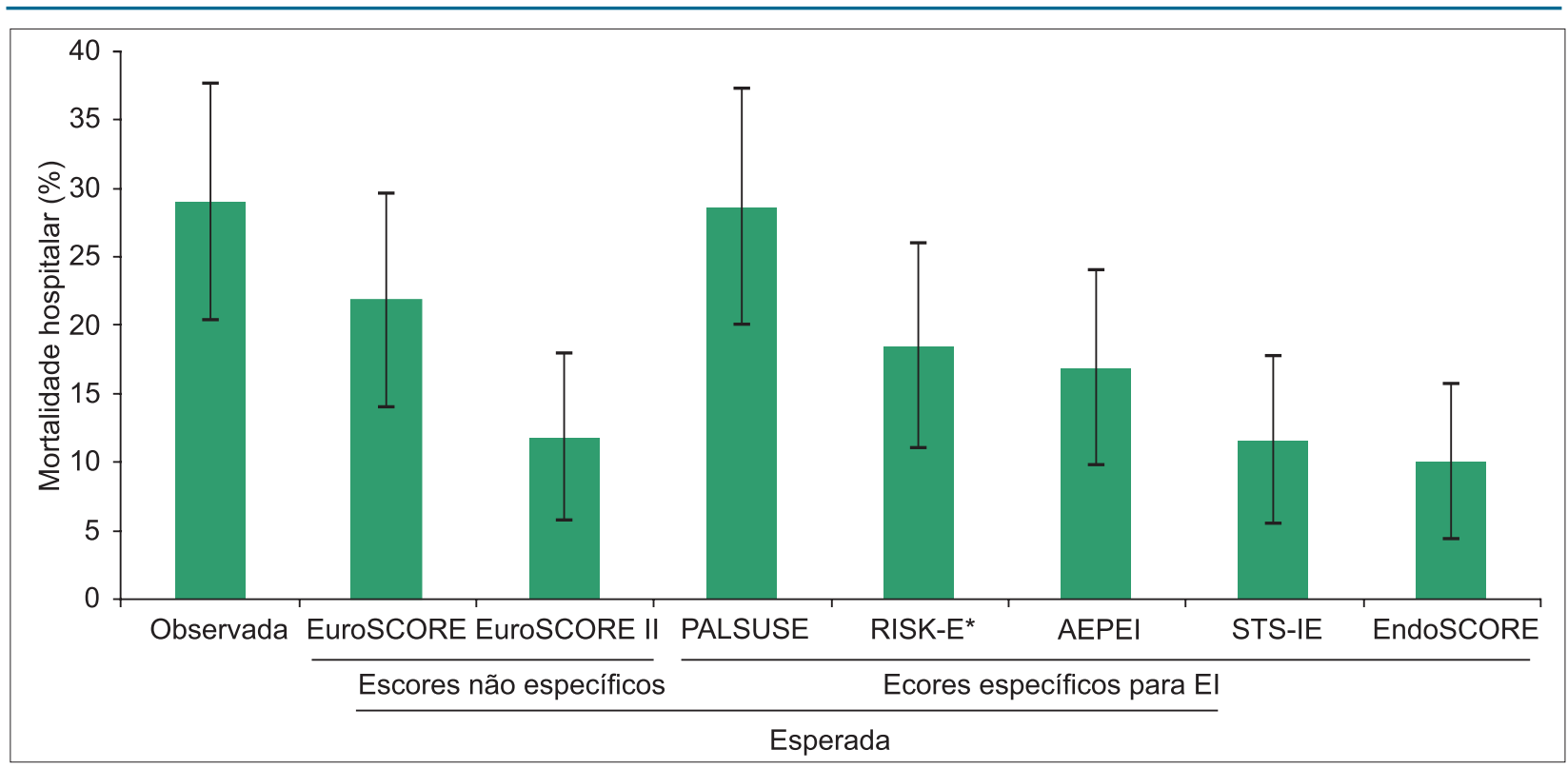

Figura 1 - Mortalidade hospitalar observada e esperada de acordo com os escores. *A mortalidade observada foi de 29,0\%, exceto para o RISK-E, que foi de $28,4 \%$ ( 5 casos de endocardite infecciosa pulmonar/tricúspide foram excluídos, uma vez que eles não estão incluídos na análise desse escore). As barras de erro indicam intervalo de confiança de $95 \%$.

Tabela 3 - Razão de mortalidade observada/esperada e análise da curva ROC para os escores estudados

\begin{tabular}{|c|c|c|c|c|c|c|}
\hline ESCORE & $\begin{array}{l}\text { MORTALIDADE } \\
\text { O/E * }\end{array}$ & IC95\% & $\mathrm{p}$ & ASC & IC95\% & $\mathrm{p}$ \\
\hline \multicolumn{7}{|c|}{ ESCORES NÃO ESPECÍFICOS PARA EI } \\
\hline $\begin{array}{l}\text { EuroSCORE } \\
\text { logístico }\end{array}$ & 1,33 & $0,92-1,87$ & 0,123 & 0,77 & $0,66-0,87$ & $<0,001$ \\
\hline EuroSCORE II & 2,46 & $1,70-3,45$ & $<0,001$ & 0,69 & $0,58-0,80$ & 0,002 \\
\hline \multicolumn{7}{|c|}{ ESCORES ESPECÍFICOS PARA EI } \\
\hline STS-IE & 2,50 & $1,73-3,50$ & $<0,001$ & 0,67 & $0,56-0,79$ & 0,005 \\
\hline PALSUSE & 1,01 & $0,70-1,42$ & 0,919 & 0,68 & $0,57-0,79$ & 0,003 \\
\hline RISK-E & 1,53 & $1,05-2,18$ & 0,029 & 0,71 & $0,60-0,81$ & 0,001 \\
\hline AEPEI & 1,71 & $1,18-2,40$ & 0,006 & 0,65 & $0,53-0,77$ & 0,017 \\
\hline EndoSCORE & 2,90 & $2,00-4,06$ & $<0,001$ & 0,76 & $0,66-0,86$ & $<0,001$ \\
\hline
\end{tabular}

*A mortalidade observada foi de 29,0\%, exceto para o RISK-E, que foi de $28,4 \%$ ( 5 casos de endocardite infecciosa pulmonarttricúspide foram excluídos, uma vez que eles não estão incluídos na análise desse escore). O/E: observada/esperada; ASC: área sob a curva; IC: intervalo de confiança; El: endocardite infecciosa.

muitas vezes, fatal, ${ }^{21}$ foi o microrganismo causador em apenas 8,4\% dos casos, ao passo que, nas coortes de validação, esse percentual variou de 17,5 a 19,9\%. ${ }^{11,12}$ Esses dois fatores provavelmente explicam, ao menos em parte, a baixa precisão dos escores específicos para El na nossa coorte. O mesmo ocorreu com outros itens incluídos nos escores específicos, tais como a classe funcional IV da NYHA no escore AEPEI ${ }^{10}$ $(37,7$ vs. $20,6 \%)$, FEVE $\leq 50 \%$ no EndoSCORE ${ }^{7}$ (35,9 vs. $15,9 \%)$, choque cardiogênico e trombocitopenia no RISK-E ${ }^{12}$ (17,9 e $29,2 \%$ no estudo original vs. 11,2 e $18,7 \%$ neste estudo, respectivamente); embora fortemente associados com a mortalidade, esses fatores não foram significativamente predominantes na nossa coorte.
O EuroSCORE II, o mais comumente utilizado para avaliação do risco pré-operatório na prática clínica atual, subestimou em 2,5 vezes a mortalidade observada e teve baixo poder discriminatório (ASC: 0,69). A coorte original do estudo EuroSCORE II tinha uma porcentagem muito baixa de pacientes com com El ativa $(2,2 \%) ;^{9}$ consequentemente, é difícil generalizar os resultados do EuroSCORE II para as populações com El. Em uma análise de 149 pacientes submetidos à cirurgia cardíaca em razão de El ativa em dois centros de referência franceses, Patrat-Delon et al., observaram que, apesar de o EuroSCORE II ter bom poder discriminatório (ASC: 0,78; IC95\%: 0,70-0,84), seus resultados deveriam ser interpretados com cautela durante a 
fase aguda da El, porque esse escore subestimou a mortalidade pós-operatória em 5-10\% na metade dos pacientes com mortalidade prevista de $>10 \%$. No Brasil, Oliveira et al., ${ }^{22}$ conduziram o único outro estudo até hoje que avaliou o uso de predição em pacientes com El ativa submetidos à cirurgia cardíaca. Nesse estudo, que incluiu 88 pacientes, o EuroSCORE II subestimou significativamente a mortalidade hospitalar, com uma razão de mortalidade O/E de 2,31 (IC95\%: 1,41-3,58; $p=0,002$ ). A análise da curva ROC não foi realizada.

Os pacientes com El ativa já haviam sido sub-representados na coorte do EuroSCORE, ${ }^{8}$ na qual a El ativa estava presente em apenas 3,6\% de todos os pacientes submetidos à cirurgia valvar. Madeira et al., ${ }^{23}$ em um estudo com 128 pacientes submetidos à cirurgia cardíaca devido a El ativa, compararam o EuroSCORE e o EuroSCORE II para predição de mortalidade perioperatória. Eles observaram que o padrão de calibração diferiu entre os dois escores: o EuroSCORE mostrou uma tendência progressiva superestimar, ao passo que o EuroSCORE II tendeu a subestimar a mortalidade. Por outro lado, assim como no presente estudo, Mestres et al., ${ }^{24}$ em um estudo com 181 pacientes com El (93,2\% ativa), descreveram bom poder discriminatório (ASC: 0,84) e uma mortalidade esperada $(27,1 \%)$ muito semelhante àquela da observada (28,8\%; razão O/E: 1,1$)$.

A necessidade de uma ferramenta de estratificação específica, útil tanto para a informação pré-operatória ao paciente quanto para a tomada de decisão à beira do leito, surge das peculiaridades da cirurgia da El em relação à cirurgia cardíaca em geral: os defechos pós-operatórios podem ser influenciados não somente por questões anatômicas ou funcionais cardiovasculares, mas também por fatores microbiológicos e infecciosos sistêmicos. ${ }^{25}$ Mais recentemente, foram desenvolvidos novos escores de risco específicos para a El. Eles incorporam alguns fatores específicos da El (tais como culturas microbiológicas, formação de abscesso e sepse), que são conhecidos preditores independentes de mortalidade. Os escores específicos para El vêm demonstrando maior precisão na predição de mortalidade do que os escores de risco tradicionais. ${ }^{26}$

Dentre os escores específicos para El analisados, apenas o PALSUSE ${ }^{10}$ e o RISK-E ${ }^{12}$ tinham coortes de derivação limitadas a pacientes com El ativa. O escore PALSUSE, ${ }^{10}$ que incorpora o EuroSCORE na sua composição, foi derivado de um estudo de coorte prospectiva com 437 pacientes que foram submetidos à cirurgia na na fase aguda da EI. Os dados foram coletados em 26 hospitais espanhóis. A mortalidade hospitalar foi de $24,3 \%$, variando de $0 \%$ em pacientes com um escore de 0 a 45,4\% naqueles com um escore $\geq 4$. A ASC foi de 0,84 (IC95\%: 0,79-0,88), indicando capacidade discriminatória satisfatória. O RISK-E ${ }^{12}$ foi desenvolvido a partir de uma pesquisa realizada em três centros de saúde terciários da Espanha, que buscavam prever a mortalidade hospitalar em 424 pacientes com El ativa aórtica/mitral submetidos à cirurgia cardíaca. A ASC foi de 0,82 (IC95\%: 0,75-0,88). A probabilidade preditiva da mortalidade pós-operatória variou de 3\% para um paciente com um escore de 0 a $97 \%$ para um paciente com o escore mais alto possível de 68. Uma comparação das ASCs apresentou desempenho preditivo superior estatisticamente significativo do RISK-E ( $p=0,01)$, em relação ao EuroSCORE, EuroSCORE II, ou PALSUSE.

De 2000 a 2015, dados de 2.715 pacientes com endocardite $(70,1 \%$ ativa) que foram submetidos à cirurgia em 26 centros cirúrgicos cardíacos italianos foram coletados retrospectivamente. Esse amplo estudo ${ }^{7}$ forneceu um modelo de risco logístico para prever a mortalidade precoce (dentro de 30 dias da cirurgia): o EndoSCORE. A ASC foi de 0,84 (IC95\%: 0,81-0,86). No nosso estudo, esse escore foi testado para prever o risco de morte durante a internação, independentemente do tempo de permanência hospitalar, e 5 das 31 mortes (16,1\%) ocorreram 30 dias após a cirurgia (mortalidade precoce: 24,3\%). Essa diferença pareceu ter pouco efeito sobre o desempenho do escore, que igualmente subestimou a mortalidade precoce (razão O/E: 2,4; ASC: 0,77 [IC95\%: 0,66-0,88]).

Embora o $\mathrm{AEPEI}^{11}$ seja um escore específico para El, ele não inclui variáveis específicas de El no seu modelo final. Ele foi desenvolvido a partir de um estudo prospectivo com 361 pacientes consecutivos que haviam sido submetidos à cirurgia em razão de El (76,2\% ativa) em oito centros cirúrgicos cardíacos europeus. Cinquenta e seis pacientes (15,5\%) morreram após a cirurgia, e a ASC foi de 0,78 (IC95\%: 0,73$0,82)$. Na população do estudo, o escore AEPEI teve poder discriminatório equivalente àquele do EuroSCORE II $(p=0,4)$ e mostrou superioridade em relação ao EuroSCORE logístico $(p=0,0026)$ e o escore PALSUSE ( $p=0,047)$.

Assim como o escore AEPEI, o STS-IE² não inclui variávies específicas para El. Ele foi desenvolvido a partir do banco de dados de cirurgia cardíaca adulta da Society of Thoracic Surgeons (STS), que foi estabelecido em 1989, incluindo dados de 3 milhões de procedimentos cardíacos de mais de $90 \%$ dos centros cirúrgicos cardíacos da América do Norte. De 2002 a 2008, 19.543 cirurgias foram realizadas em pacientes com El (51,5\% ativa), com uma mortalidade de $8,2 \%$. O STS-IE demonstrou boa capacidade preditiva, com uma ASC de 0,76.

Algumas limitações do nosso estudo devem ser mencionadas. Em primeiro lugar, o desenho retrospectivo pode ter influenciado a qualidade e a consistência dos dados coletados. O tamanho da amostra relativamente pequeno também é uma fonte de preocupação. Finalmente, o fato de o estudo ter sido conduzido em um único centro pode limitar a validade externa dos nossos achados.

\section{Conclusões}

Nossos resultados mostraram que, apesar da disponibilidade de escores recentes específicos para El, e considerando-se o equilíbrio entre os índices, o EuroSCORE logístico pareceu ser o melhor preditor de risco de mortalidade na nossa coorte de pacientes com $\mathrm{El}$, admitidos durante um período de 10 anos, levando-se em conta a calibração (razão O/E: 1,3) e a capacidade discriminante (ASC: 0,77). Esse achado tem implicações clínicas, já que o EuroSCORE II é o escore mais frequentemente utilizado na avaliação pré-operatória. Faz-se necessária a validação local de escores específicos para avaliar o risco pré-operatório dos pacientes com El. 


\section{Contribuição dos Autores}

Concepção e desenho da pesquisa, análise e interpretação dos dados e redação do manuscrito: Pivatto Júnior F, Gus M; Obtenção de dados: Pivatto Júnior F, Bellagamba CCA, Fernandes FS, Butzke M, Busato SB; Análise estatística e obtenção de financiamento: Pivatto Júnior F; Revisão crítica do manuscrito quanto ao conteúdo intelectual importante: Pivatto Júnior F, Bellagamba CCA, Pianca EG, Fernandes FS, Butzke M, Busato SB, Gus M.

\section{Potencial Conflito de Interesses}

Declaro não haver conflito de interesses pertinentes.

\section{Fontes de Financiamento}

O presente estudo foi financiado pelo Fundo de Incentivo à Pesquisa e Eventos (FIPE) do Hospital de Clínicas de Porto Alegre (HCPA).

\section{Vinculação Acadêmica}

Não há vinculação deste estudo a programas de pós-graduação.

\section{Aprovação Ética e Consentimento Informado}

Este estudo foi aprovado pelo Comitê de Ética do Hospital de Clínicas de Porto Alegre sob o número de protocolo 2016-0632. Todos os procedimentos envolvidos nesse estudo estão de acordo com a Declaração de Helsinki de 1975, atualizada em 2013.

\section{Referências}

1. Kang DH, Kim YJ, Kim SH, Sun BJ, Kim DH, Yun SC, et al. Early surgery versus conventional treatment for infective endocarditis. N Engl J Med. 2012;366:2466-73.

2. Gaca JG, Sheng S, Daneshmand MA, O'Brien S, Rankin JS, Brennan JM, et al. Outcomes for endocarditis surgery in North America: a simplified risk scoring system. J Thorac Cardiovasc Surg. 2011;141(1):98-106.e2.

3. Cahill TJ, Baddour LM, Habib G, Hoen B, Salaun E, Pettersson GB, et al. Challenges in infective endocarditis. J Am Coll Cardiol. 2017;69(3):325-44.

4. Que YA, Moreillon P. Infective endocarditis. Nat Rev Cardiol. 2011;8(6):322-36.

5. Habib G, Lancellotti P, Antunes MJ, Bongiorni MG, Casalta JP, Del Zotti F, etal. 2015 ESC Guidelines for the management of infective endocarditis: The task force for the management of infective endocarditis of the European Society of Cardiology (ESC)Endorsed by: European Association for Cardio-Thoracic Surgery (EACTS), the European Association of Nuclear Medicine (EANM). Eur Heart J. 2015;36(44):3075-128

6. Patrat-Delon S, Rouxel A, Gacouin A, Revest M, Flécher E, Fouquet O, et al. EuroSCORE II underestimates mortality after cardiac surgery for infective endocarditis. Eur J Cardiothorac Surg. 2016;49(3):944-51.

7. Di Mauro M, Dato GMA, Barili F, Gelsomino S, Santè P, Corte AD, et al. A predictive model for early mortality after surgical treatment of heart valve or prosthesis infective endocarditis. The EndoSCORE. Int J Cardiol. 2017 Aug $15 \cdot 241 \cdot 97-102$

8. Roques F, Nashef SA, Michel P, Gauducheau E, de Vincentiis C, Baudet E, et al. Risk factors and outcome in European cardiac surgery: analysis of the EuroSCORE multinational database of 19030 patients. Eur J Cardiothorac Surg. 1999;15(6):816-22.

9. Nashef SA, Roques F, Sharples LD, Nilsson J, Smith C, Goldstone AR, et al. EuroSCORE II. Eur J Cardiothorac Surg. 2012;41(4):734-44.

10. Martínez-Sellés M, Muñoz P, Arnáiz A, Moreno M, GálvezJ, Rodríguez-Roda $\mathrm{J}$, et al. Valve surgery in active infective endocarditis: a simple score to predict in-hospital prognosis. Int J Cardiol. 2014;175(1):133-7.

11. Gatti G, Perrotti A, Obadia J, Duval X, lung B, Alla F, et al. Simple scoring system to predict in-hospital mortality after surgery for infective endocarditis. JAm Heart Assoc. 2017;6(7):pii:e004806

12. Olmos C, Vilacosta I, Habib G, Maroto L, Fernández C, López J, et al. Risk score for cardiac surgery in active left-sided infective endocarditis. Heart. 2017;103(18):1435-42.

13. Li JS, Sexton DJ, Mick N, Nettles R, Fowler VG, Ryan T, et al. Proposed modifications to the Duke Criteria for the diagnosis of infective endocarditis. Clin Infect Dis. 2000;30(4):633-8.

14. Cockcroft DW, Gault H. Prediction of creatinine clearance from serum creatinine. Nephron. 1976;16(1):31-41.

15. Summary of recommendation statements. Kidney Int Suppl. 2013;3(1):5-14

16. Sullivan KM, Dean AG, Soe MM. OpenEpi: a web-based epidemiologic and statistical calculator for public health. Public Health Rep. 2009;124(3):471-4.

17. Søreide K. Receiver-operating characteristic curve analysis in diagnostic prognostic and predictive biomarker research. J Clin Pathol. 2009 Jan;62(1):1-5.

18. Hajian-Tilaki K. Receiver Operating Characteristic (ROC) curve analysis for medical diagnostic test evaluation. Caspian J Intern Med. 2013;4(2):627-35.

19. Nezic D, Spasic T, Micovic S, Kosevic D, Petrovic I, Lausevic-Vuk L, et al. Consecutive observational study to validate EuroSCORE II performances on a single-center, contemporary cardiac surgical cohort. J Cardiothorac Vasc Anesth. 2016;30(2):345-51.

20. Nashef SAM. Death and quality in cardiac surgery. J Patient Saf Risk Manag 2010;16(4):130-4.

21. Fernández Guerrero ML, González López JJ, Goyenechea A, Fraile J, de Górgolas M. Endocarditis caused by Staphylococcus aureus: a reappraisa of the epidemiologic, clinical, and pathologic manifestations with analysis of factors determining outcome. Medicine (Baltimore). 2009;88(1):1-22.

22. Oliveira JLR, Santos MAD, Arnoni RT, Ramos A, Togna DD, Ghorayeb SK, et al. Mortality predictors in the surgical treatment of active infective endocarditis. Braz J Cardiovasc Surg. 2018;33(1):32-9.

23. Madeira S, Rodrigues R, Tralhão A, Santos M, Almeida C, Marques M, et al. Assessment of perioperative mortality risk in patients with infective endocarditis undergoing cardiac surgery: performance of the EuroSCORE and II logistic models. Interact Cardiovasc Thorac Surg. 2016;22(2):141-8.

24. Mestres CA, Castro MA, Bernabeu E, Josa M, Cartaňá R, Pomar JL, et al. Preoperative risk stratification in infective endocarditis. Does the EuroSCORE model work? Preliminary results. Eur J Cardiothorac Surg. $2007 ; 32(2): 281-5$.

25. Della Corte A, Cotrufo M, Carozza A. Endocarditis surgery: need for a specific risk scoring system. J Thorac Cardiovasc Surg. 2011;142(3):721.

26. Varela L, López-Menéndez J, Redondo A, Fajardo ER, Miguelena J, Centella T, et al. Mortality risk prediction in infective endocarditis surgery: reliability analysis of specific scores. Eur J Cardiothorac Surg. 2018;53(5):1049-54. 